\title{
Significant correlation between micrometastasis in the lymph nodes and reduced expression of E-cadherin in early gastric cancer
}

\author{
Jianhui Cai ${ }^{1,2}$, Masahide Ikeguchi ${ }^{2}$, Shunichi Tsujitani ${ }^{2}$, Michio Maeta ${ }^{3}$, Jin Liu ${ }^{1,2}$, and Nobuaki Kaibara ${ }^{2}$ \\ ${ }^{1}$ Department of Surgery, Second Affiliated Hospital of Hebei Medical University, Hebei, China \\ ${ }^{2}$ First Department of Surgery, Faculty of Medicine, Tottori University, 36-1 Nishichou, Yonago 683-8504, Japan \\ ${ }^{3}$ Department of Fundamental Nursing, School of Health Science, Tottori University, 36-1 Nishichou, Yonago 683-8504, Japan
}

\begin{abstract}
Background. E-cadherin has been recognized as an important factor associated with tumor metastasis. However, the relationship between micrometastasis in the lymph nodes and the expression of E-cadherin in the primary tumor in gastric cancer remains unclear.

Methods. Two consecutive sections of $\mathbf{4 5 2 2}$ lymph nodes from 162 patients with early gastric cancer were prepared for simultaneous hematoxylin and eosin $(\mathrm{H} \& \mathrm{E})$ and cytokeratin $(\mathrm{CK})$ staining. Sections of primary tumors from 135 of these patients were prepared for $\mathbf{E}$-cadherin immunostaining.

Results. The incidence of lymph node involvement was significantly increased, from $6.8 \%$ (11/162 patients) by $\mathrm{H \& E}$ staining, to $27 \%$ (43/162 patients) by CK immunostaining $(P<$ 0.0001). Micrometastasis in the lymph node was found in 32 of $151(21 \%)$ patients who had no lymph node metastasis evidenced by $\mathrm{H} \& \mathrm{E}$ staining. Micro-lymph node metastasis was frequently found in tumors with a diameter more than $1.0 \mathrm{~cm}$, of those that were poorly differentiated, deeply invaded, showed lymphatic on vascular invasion, and in those that showed reduced expression of E-cadherin. Loss of expression of E-cadherin in the primary tumor was closely correlated with micro-lymph node metastasis. Patients with tumors with micro-lymph node metastasis detected by CK immunostaining had a significantly lower 5-year survival rate $(P<0.01)$ than those without such metastases.

Conclusion. Tumors more than $1.0 \mathrm{~cm}$ in diameter and those that exhibit poor differentiation, deep invasion (i.e., to the submucosa), lymphatic or vascular invasion, and reduced expression of E-cadherin are risk factors for lymph node metastasis in early gastric cancer. Thus, it is recommended that cancers confined to the mucosa (m-cancers) that are more than $1.0 \mathrm{~cm}$ in diameter should not be treated with limited surgery without lymphadenectomy.
\end{abstract}

Key words Early gastric cancer - Micrometastasis Cytokeratin · E-cadherin · Immunohistochemistry

\section{Introduction}

Early gastric cancer (EGC) is defined as cancer that is confined to the mucosa (m-cancer) or submucosa (smcancer), regardless of the presence or absence of regional lymph node metastasis [1]. Recent improvements in endoscopic techniques and the widespread use of mass screening have made it possible to detect gastric cancer at a very early stage, and have led to excellent outcomes for patients after surgical treatment [2-4]. Although gastrectomy with D2 lymphadenectomy has been the standard treatment for early gastric cancer [5-7], a less invasive surgerical prodecure without lymphadenectomy has recently been used to treat these patients and has achieved not only complete cure of the disease but also a better postoperative quality of life $[8,9]$.

It is well known that lymph node metastasis is the most important prognostic factor for patients with early gastric cancer $[10,11]$. Moreover, micrometastasis in the lymph node has been detected by cytokeratin (CK) immunostaining [12-14]. The clinicopathological significance of these so-called micrometastases in the lymph nodes and the correlation of these micrometastases with the reduced expression of E-cadherin (E-cad), which has been recognized as an important factor in tumor metastasis [15], have not been extensively discussed. Because it is still sometimes difficult to predict lymph node involvement preoperatively, a better understanding of the clinicopathological characteristics of micrometastasis in the lymph node is important for the establishment of a therapy for early gastric cancer. Accordingly, in the present study, we examined the relationship between micrometastasis in lymph nodes and the expression of E-cad, in patients with EGC. 


\section{Patients and methods}

\section{Patients}

A total of 162 patients with EGC who underwent curative gastrectomy at Tottori University Hospital from 1986 to 1990 were investigated. There were 98 men and 64 women, ranging in age from 37 to 82 years, with a mean age of 63 years. Total gastrectomy was performed in 19 patients (12\%), distal subtotal gastrectomy in 126 patients $(78 \%)$, proximal subtotal gastrectomy in 15 patients $(9.3 \%)$, and limited resection in 2 patients $(1.2 \%)$. Two patients underwent D1 lymphadenectomy (removal of perigastric nodes; group 1), 115 patients underwent D2 lymphadenectomy (D1 plus removal of the nodes along the left gastric, common hepatic, celiac, and splenic arteries; group 2), and 45 patients underwent D2 plus a part of group 3 lymphadenectomy (lymph nodes in the hepatoduodenal ligament, behind the pancreas head, and at the root of the mesentery). All patients were followed for at least 5 years after surgery.

\section{Clinicopathological data}

Clinicopathological parameters were evaluated according to the General rules for gastric cancer study in surgery and pathology proposed by the Japanese Research Society for Gastric Cancer [1]. Tumor size was determined on the basis of the superficial maximum diameter of the primary lesions. Macroscopic types were described as 0-I, protruded; 0-IIa, superficial elevated; 0-IIb, flat; 0-IIc, superficial depressed; and 0-III, excavated. Histologically, 107 tumors were classified as differentiated carcinomas (51 well differentiated and 57 moderately differentiated carcinomas). Fifty-five tumors were classified as poorly differentiated carcinomas. The depth of invasion of the tumors, as determined by $H \& E$ staining, was classified into four subgroups: intramucosal cancer (tumor invasion limited to mucosa; m-cancer; $n=84$ ), submucosal 1 cancer (slight invasion, limited to the upper two-thirds of the submucosa; sm1; $n=49$ ), and submucosal 2 cancer (deep submucosal invasion close to the muscularis properia; $\operatorname{sm} 2 ; n=29$ ). Lymphatic invasion was detected in 15 patients, and vascular invasion in 14 patients.

\section{Cytokeratin immunostaining of lymph nodes}

A total of 4522 lymph nodes from the 162 patients with EGC were sampled, with a median number of 28 nodes (range, 10-61 nodes) per patient. Two consecutive sections, each $4-\mu \mathrm{m}$-thick, were prepared from the lymph nodes for simultaneous staining with ordinary $H \& E$ and CK immunostaining. Forty perigastric lymph nodes, obtained from 20 patients with benign gastric ulcers, were used as normal controls, and 135 main tumors were used as positive controls. Mouse monoclonal immunoglobulin, CAM 5.2 (Becton Dickinson, San Jose, CA, USA), was used as a primary antibody. A standard streptavidin-biotin (SAB) method was used for CK immunostaining, as previously described [16]. Briefly, the sections were dewaxed and dehydrated. Endogenous peroxidase was blocked by incubation of the samples with 3\% hydrogen peroxide in 100\% methanol. The tissue sections were incubated with the primary antibody, CAM 5.2, at $25 \mu \mathrm{g}$ per $\mathrm{ml}$, overnight at $4{ }^{\circ} \mathrm{C}$. The second antibodies, biotinylated antibodies against mouse immunoglobulin, were applied, followed by the application of peroxidase-labeled streptavidin. The reaction products were visualized with diaminobenzidine as the chromogen, and sections were counterstained with methyl green. Tris-buffered saline was used instead of the primary antibody as a negative control.

\section{E-cadherin immunostaining of primary tumors}

Sections were prepared from 135 primary tumors of the 162 patients with EGC; 27 primary tumors were excluded because the samples were too small to be cut into sections again. Mouse anti-human E-cadherin monoclonal antibody, HECD-1 (Diluted 1:400; Takara, Otsu, Japan), was used as the primary antibody. A standard avidin biotin penoxidase complex (ABC) technique was performed, using a Vectastain $\mathrm{ABC}$ Kit (Vector Laboratories, Burlingame, CA, USA), as described previously $[17,18]$. Phosphate-buffered saline (PBS) was used instead of the primary antibody as a negative control. Adjacent noninvolved gastric mucosa was used as an internal positive control.

\section{Interpretation of the immunostaining}

The H\&E-stained slides, at a magnification of 200, were first assessed by an experienced pathologist (M.M.) for the presence of a metastasis in the lymph node. The CKimmunostained slides were then examined, and the results were compared with those obtained from the H\&E slides. Micrometastasis in the lymph node was recognized when tumor cells were detected only by CK immunostaining, and were not detected by H\&E staining. E-cad immunostaining was scored in a semiquantitative fashion, from 0 to 3 [19], with 0 denoting absent staining (normal staining less than $10 \%$ of the tumor areas), 1 denoting cytoplasmic distribution, 2 denoting heterogeneous staining (normal staining in 10\% to $90 \%$ of the tumor area), and 3 denoting a normal membranous staining pattern (normal staining in more than $90 \%$ of the tumor area). For the purpose of data 
analysis, the absent, cytoplasmic, and heterogeneous staining patterns (i.e., scores 0,1 , and 2 ) were classified as a loss of or reduced expression of E-cad. Tumors with a normal membranous pattern that was similar to that in the adjacent normal mucosa (i.e., score of 3) were classified as having preserved expression of E-cad. All the staining results for $\mathrm{CK}$ and $\mathrm{E}$-cad were examined in relation to the clinicopathological parameters of the tumors.

\section{Statistical analysis}

The $\chi^{2}$ test was used for correlating lymph node involvement and antigen expression with clinicopathologic indices. Survival curves were constructed according to the method of Kaplan-Meier; for differences between curves, the $P$ value was calculated using the generalized Wilcoxon test. A multivariate Cox regression model was used to examine the risk factors associated with nodal involvement and patients' survival. A $P$ value of less than 0.05 was considered an indication of statistical significance.

\section{Results}

\section{Lymph node involvement in EGC}

The incidence of lymph node involvement was found to be $6.8 \%$ (11/162 patients) according to $\mathrm{H} \& \mathrm{E}$ staining, with $1(1.2 \%)$ of the 84 patients with $\mathrm{m}$-cancer and 10 $(13 \%)$ of the 78 patients with sm-cancer. However, the incidence of lymph node involvement was found to be $27 \%$ (43/162 patients) according to CK-immunostaining $(P<0.0001)$, with $16(19 \%)$ of the 84 patients with $\mathrm{m}$ cancer and $27(35 \%)$ of the 78 patients with sm-cancer. With regard to the total number of dissected lymph nodes, the frequency of lymph node involvement was significantly increased, from $0.5 \%$ (22/4522 nodes) detected by H\&E staining, to $2.5 \%$ (112/4522 nodes) detected by $\mathrm{CK}$ immunostaining $(P<0.0001)$. The incidence of micrometastasis in lymph nodes with no evidence of metastasis according to $\mathrm{H} \& \mathrm{E}$ staining was $21 \%$ (32/151 patients). Twelve ( $75 \%$ ) of the $16 \mathrm{~m}$-cancers that were node-positive were found to consist of only single or scattered cancer cells in the lymph nodes. On the other hand, metastases, in the form of scattered or clustered cancer cells in the lymph nodes, were detected in $19(70 \%)$ of the $27 \mathrm{sm}$-cancers that were nodepositive (Fig. 1, a, b, c). Nineteen (44\%) of the 43 patients with node-positive cancer were found to have involvement of the extra-perigastric nodes; 16 patients with involvement in group 2 nodes and 3 patients with involvement in group 3 nodes. No CK-positive cells were detected in any of the 40 nodes from patients with benign gastric ulcers.

\section{Clinicopathological significance of lymph node involvement}

We analyzed clinicopathologic factors in the 43 patients with lymph node involvement according to $\mathrm{CK}$ immunostaining (Table 1). Lymph node involvement was detected more frequently in tumors with a diameter of more than $2.0 \mathrm{~cm}$ compared with tumors of less than 1$\mathrm{cm}$ diameter $(P<0.05)$. Twenty-three $(85 \%)$ of the 27 sm-cancers with lymph node metastasis detected by CK immunostaining were found to be more than $2.0 \mathrm{~cm}$ in diameter, while 4 (15\%) of these sm-cancers were found to be $2.0 \mathrm{~cm}$ or less in diameter and 3 of these 4 had metastasized to the extra-perigastric (group 2) lymph nodes (Table 2). Six (38\%) of the $16 \mathrm{~m}$-cancers that were CK-positive in the lymph nodes were found to be $2.0 \mathrm{~cm}$ or less in diameter, with 5 of them being 1.1$2.0 \mathrm{~cm}$ in diameter, although all of these micrometastases were only single cancer cells in the perigastric (group 1) lymph nodes. In all 43 patients with CKpositive lymph nodes, metastases, in the form of scattered or clustered cancer cells in the lymph node, occurred more frequently in tumors with a diameter of more than $2 \mathrm{~cm}$. As shown in Table 1, poorly differentiated tumors (shown as "undifferentiated" in Table 1), deeply invaded tumors (i.e., to the submucosa), and tumors with lymphatic or vascular invasion were strongly associated with lymph node involvement. In addition, $16(62 \%)$ of 26 tumors with discrete or clustered cancer cells in the lymph node were poorly differentiated carcinomas. Moreover, 12 (60\%) of 20 undifferentiated tumors were found to have extraperigastric lymph node metastases (group 2 and/or 3).

\section{Expression of E-cadherin}

E-cad was strongly expressed at the cell-cell boundaries of normal gastric mucosa adjacent to the tumor. Loss of expression of E-cad was found in $77(57 \%)$ of the 135 patients with EGC examined for E-cad (Fig. 2a,b). Expression of E-cad was significantly reduced in tumors with a diameter of more than $1.0 \mathrm{~cm}(P<0.05$ vs $\leq 1.0 \mathrm{~cm})$, undifferentiated tumors $(P<0.0001)$, deeply invaded tumors $(P<0.005)$, and tumors with lymphatic invasion and vascular invasion $(P<0.05$ vs no invasion; Table 3$)$. As shown in Table 4, 30 (81\%) of the 37 cases with CK-positive lymph nodes had loss of E-cad expression $(P<0.0005)$. A high incidence of loss of E-cad expression was found not only in the tumors with clustered cancer cells in the lymph nodes but also in those with single or scattered cancer cells in the lymph nodes. Moreover, the tumors with loss of E-cad expression had a large number of metastatic lymph nodes and a large number of extra-perigastric metastatic lymph nodes (Table 4). 

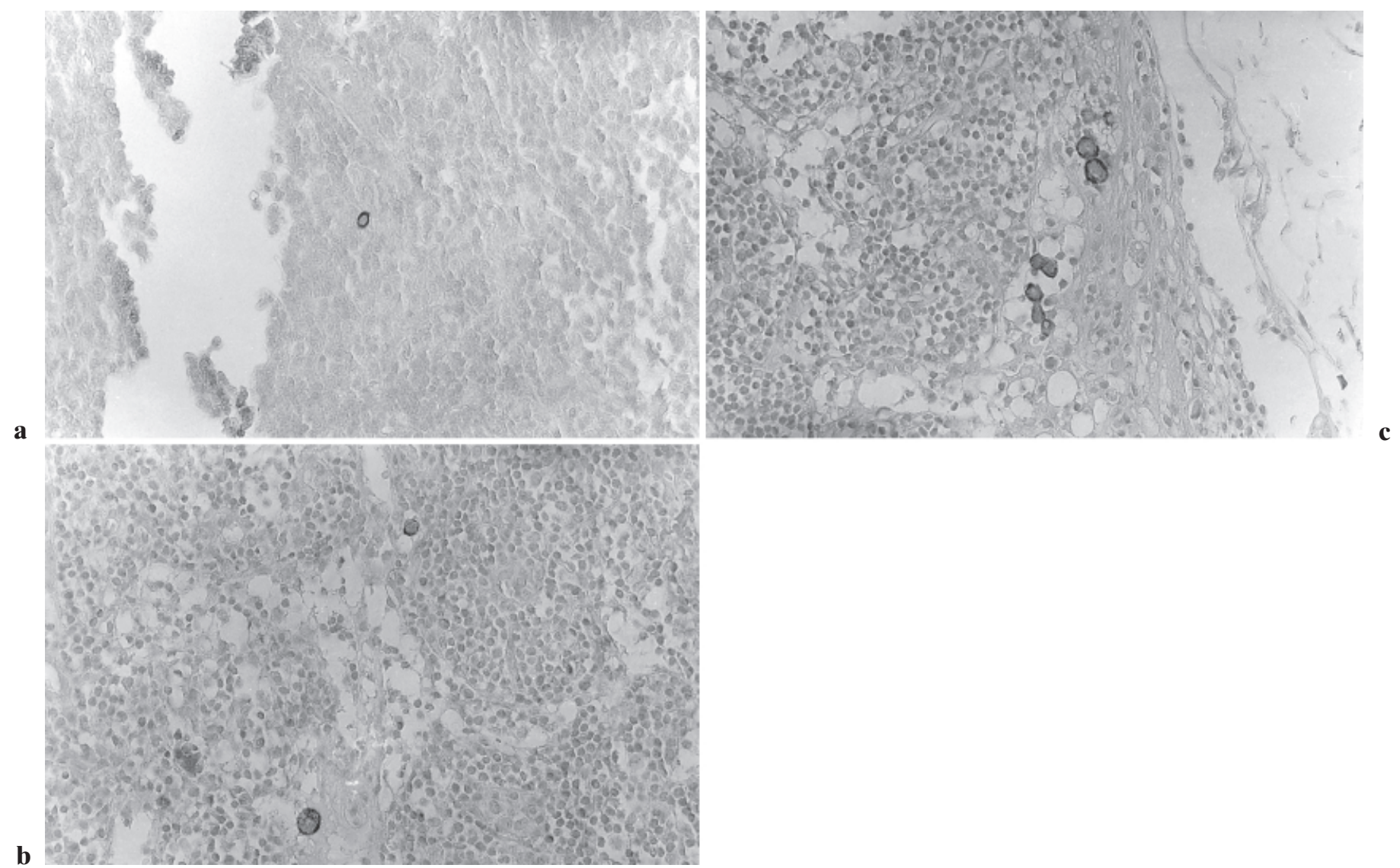

Fig. 1a-c. Micrometastases in lymph nodes, detected by cytokeratin (CK) immunostaining, that had been overlooked by ordinary H\&E staining. $\times 200$. a A single cancer cell in the lymph node $\mathbf{b}$ Scattered cancer cells in the lymph node $\mathbf{c}$ Clustered cancer cells in the lymph node

\section{Prognosis of patients with EGC}

The overall 5-year survival rate of our 162 patients with EGC was $98 \%$. Four patients died of a postoperative recurrence of the tumor ( 1 of liver metastasis and 3 of peritoneal and lymph node metastasis). Two of these patients had already been diagnosed with lymph node metastases by H\&E staining immediately after the operation. In another 2 patients, the lymph nodes were diagnosed as micrometastasis-positive by CK immunostaining, although this condition had not been detected by $H \& E$ staining. In comparison with the 119 patients without lymph node involvement (5-year survival rate of $100 \%$ ), the 32 patients with CK-positive lymph nodes who were H\&E-negative (5-year survival rate of 93.6\%) and the 11 patients with $\mathrm{H} \& \mathrm{E}-$ positive lymph nodes (5-year survival rate of $81.8 \%$ ) had a significantly less favorable outcome $(P<0.01$ respectively; Fig. 3$)$.

By multivariate regression analysis, lymphatic invasion and abnormal expression of E-cad were found to be independent risk factors for lymph node metastasis as detected by $\mathrm{CK}$ immunostaining $(P<0.05$, respectively; Table 5). Multivariate survival analysis indicated that macroscopic type and ulceration of the tumor, but not lymph node involvement, were of independent prognostic significance $(P<0.05$, respectively).

\section{Discussion}

It has been widely recognized that large tumor size, lymphatic invasion, and deep invasion (to the submucosa) are important risk factors for lymph node metastasis $[2,3]$. In the present CK-immunohistochemical study, tumor size, deep invasion to the submucosa, lymphatic invasion, vascular invasion, and poor differentiation of the tumor were five important risk factors correlated with lymph node involvement in EGC detected by CK-immunostaining. Histologically, metastasis to the lymph node was extremely rare in tumors with a diameter of less than $2 \mathrm{~cm}[2,7]$. We also found that tumors with a diameter of more than $2 \mathrm{~cm}$ more frequently metastasized to the lymph nodes, and most of these involvements were in the form of clusters of cancer cells in the lymph nodes. However, in five m-cancers 
Table 1. Lymph node involvement and clinicopathological characteristics of the primary tumor in 162 patients with EGC

\begin{tabular}{|c|c|c|c|}
\hline Variables $^{\mathrm{a}}$ & Total no. of cases & $\begin{array}{c}\text { Number of } \\
\text { CK-positive cases }(\%)\end{array}$ & $P$ \\
\hline \multicolumn{4}{|c|}{ Superficial diameter $(\mathrm{cm})$} \\
\hline$\leq 1.0$ & 18 & $1(6)$ & \multirow{3}{*}{0.0472} \\
\hline $1.0-2.0$ & 41 & $9(22)$ & \\
\hline$>2.0$ & 103 & $33(32)$ & \\
\hline \multicolumn{4}{|l|}{ Macroscopic type } \\
\hline I, IIa, IIa + I & 23 & $4(17)$ & \multirow{3}{*}{0.1294} \\
\hline $\mathrm{IIa}+\mathrm{II}$, IIc + IIa & 27 & $10(37)$ & \\
\hline IIc, III, IIc+III & 112 & $29(26)$ & \\
\hline \multicolumn{4}{|l|}{ Ulcer formation } \\
\hline Negative & 143 & $36(25)$ & \multirow[t]{2}{*}{0.2792} \\
\hline Positive & 19 & $7(37)$ & \\
\hline \multicolumn{4}{|l|}{ Histopathology } \\
\hline Differentiated & 107 & $23(21)$ & \multirow[t]{2}{*}{0.0424} \\
\hline Undifferentiated & 55 & $20(36)$ & \\
\hline \multicolumn{4}{|l|}{ Depth of invasion } \\
\hline $\mathrm{m}$ & 84 & $16(19)$ & \multirow{3}{*}{0.0005} \\
\hline $\operatorname{sm} 1$ & 49 & $11(22)$ & \\
\hline $\operatorname{sm} 2$ & 29 & $16(55)$ & \\
\hline \multicolumn{4}{|l|}{ Lymphatic invasion } \\
\hline Negative & 147 & $33(22)$ & \multirow[t]{2}{*}{0.0002} \\
\hline Positive & 15 & $10(67)$ & \\
\hline \multicolumn{4}{|l|}{ Vascular invasion } \\
\hline Negative & 148 & $36(24)$ & \multirow[t]{2}{*}{0.0376} \\
\hline Positive & 14 & $7(50)$ & \\
\hline
\end{tabular}

EGC, Early gastric cancer; CK, cytokeratin (immunostaining)

${ }^{\text {a }}$ Evaluated according to the General rules for gastric cancer study in surgery and pathology [1]

Table 2. Correlation between lymph node metastases detected by CK immunostaining and tumor size

\begin{tabular}{|c|c|c|c|c|c|c|c|}
\hline \multirow{2}{*}{$\begin{array}{l}\text { Depth of } \\
\text { invasion }\end{array}$} & \multirow[b]{2}{*}{$n$} & \multicolumn{2}{|c|}{$\leq 1.0 \mathrm{~cm}$} & \multicolumn{2}{|c|}{$1.1-2.0 \mathrm{~cm}$} & \multicolumn{2}{|c|}{$>2.0 \mathrm{~cm}$} \\
\hline & & Perigastric & Extraperi. $^{a}$ & Perigastric & Extraperi. $^{\mathrm{a}}$ & Perigastric & Extraperi. ${ }^{a}$ \\
\hline m-cancer & 16 & 1 & 0 & 5 & 0 & 5 & 5 \\
\hline sm-cancer & 27 & 0 & 0 & 1 & 3 & 12 & 11 \\
\hline
\end{tabular}

${ }^{\mathrm{a}}$ Extra-perigastric lymph nodes

and four sm-cancers, micrometastases were found with tumors 1 to $2 \mathrm{~cm}$ in diameter, although all of these micrometastases were of only single or scattered cancer cells in the lymph nodes.

It is interesting to note that all five of the m-cancers had only perigastric lymph node involvement, but three of the four sm-cancers had an extra-perigastric lymph node metastasis. This finding strongly agrees with a recent report by Yasuda et al. [20], which also suggested the histological possibility of metastasis in a tumor with a diameter of 1 to $2 \mathrm{~cm}$. These findings indicate that an EGC with a diameter of more than $1.0 \mathrm{~cm}$ seems to be a risk for lymph node metastasis.

It is widely accepted that tumors with deep invasion or lymphatic invasion are more likely to involve lymph node metastasis [21-23]. We found that the incidence of lymph node metastasis detected by CK immunostaining was $19 \%$ in $\mathrm{m}$-cancers but $35 \%$ in sm-cancers. Moreover, most of the metastases detected from m-cancers were only single or scattered cancer cells in the lymph nodes, while, in comparison, most of those detected from sm-cancers were scattered or clustered cancer cells in the lymph nodes. It seems that deeper invasion indicates a greater number of cancer cells metastasizing to the lymph nodes. Moreover, in the current study, $67 \%$ of the patients with lymphatic invasion were found to have cancer-positive lymph nodes. Multivariate analysis showed that lymphatic invasion was an independent risk factor for lymph node metastasis. The existence and abundance of lymphatic vessels in the submucosal layer of the gastric wall may facilitate tumor metastasis $[22,24]$. These findings indicate that tumors that have 

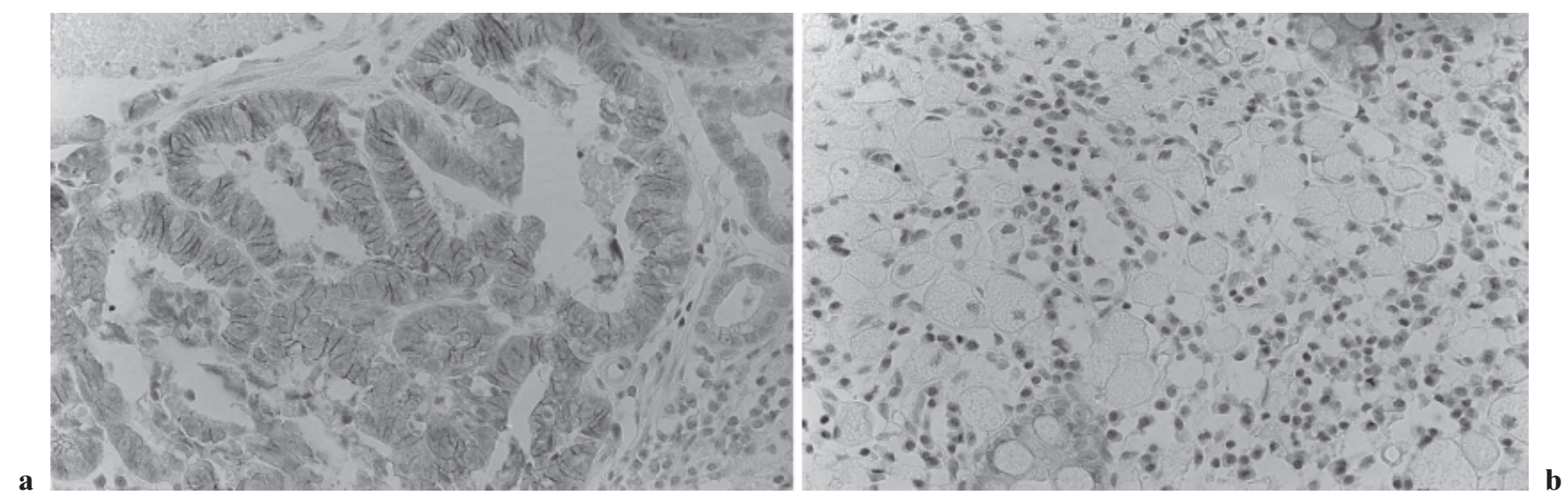

Fig. 2a,b. Expression of E-cadherin in cell-cell boundaries of early gastric cancer cells. $\times 200$. a Preserved expression $\mathbf{b}$ Loss of expression

Table 3. Relationship between clinicopathological parameters and reduced expression of E-cadherin in 135 patients with EGC tested for E-cadherin expression

\begin{tabular}{|c|c|c|c|}
\hline Clinicopathological parameters & $\begin{array}{l}\text { No. of } \\
\text { cases }\end{array}$ & $\begin{array}{c}\text { Loss of expression of } \\
\text { E-cadherin }(\%)\end{array}$ & $P$ \\
\hline \multicolumn{4}{|l|}{ Tumor size $(\mathrm{cm})$} \\
\hline$\leq 1.0$ & 14 & $2(14)$ & \multirow{3}{*}{0.0005} \\
\hline $1.1-2.0$ & 33 & $16(48)$ & \\
\hline$>2.0$ & 88 & $59(67)$ & \\
\hline \multicolumn{4}{|l|}{ Macroscopic type } \\
\hline $\mathrm{I}, \mathrm{IIa}, \mathrm{IIa}+\mathrm{I}$ & 20 & $8(40)$ & \multirow{3}{*}{0.2489} \\
\hline $\mathrm{IIa}+\mathrm{IIc}, \mathrm{IIc}+\mathrm{IIa}$ & 25 & $15(60)$ & \\
\hline IIc, III, IIc+III & 90 & $54(60)$ & \\
\hline \multicolumn{4}{|l|}{ Ulcer formation } \\
\hline Negative & 118 & $64(54)$ & \multirow[b]{2}{*}{0.0834} \\
\hline Positive & 17 & $13(76)$ & \\
\hline \multicolumn{4}{|l|}{ Histopathology } \\
\hline Differentiated & 90 & 37 (41) & \multirow[b]{2}{*}{$<0.0001$} \\
\hline Undifferentiated & 45 & $40(89)$ & \\
\hline \multicolumn{4}{|l|}{ Depth of invasion } \\
\hline $\mathrm{m}$ & 75 & $36(48)$ & \multirow{3}{*}{0.0020} \\
\hline sm1 & 35 & $19(54)$ & \\
\hline $\operatorname{sm} 2$ & 25 & $22(88)$ & \\
\hline \multicolumn{4}{|l|}{ Lymphatic invasion } \\
\hline Negative & 123 & $66(54)$ & \multirow[t]{2}{*}{0.0111} \\
\hline Positive & 12 & $11(92)$ & \\
\hline \multicolumn{4}{|l|}{ Vascular invasion } \\
\hline Negative & 122 & $66(54)$ & \multirow[t]{2}{*}{0.0346} \\
\hline Positive & 13 & $11(85)$ & \\
\hline \multicolumn{4}{|l|}{ CK staining in lymph nodes } \\
\hline Negative & 98 & $47(48)$ & \multirow[t]{2}{*}{0.0005} \\
\hline Positive & 37 & $30(81)$ & \\
\hline
\end{tabular}

invaded to the submucosa may easily intrude into the lymphatic system and further metastasize to the lymph nodes. Because deeply invaded sm-cancer has a high risk of lymph node metastasis, lymphadenectomy should be recommended for these tumors.

The E-cadherin gene has generally been recognized as an invasion-suppressor gene [15,25]. E-cadherin
(E-cad) plays a key role in the establishment and maintenance of epithelial tissue structure, and its downregulation is potentially important in the formation of metastases from carcinomas [26,27]. To elucidate the correlation between the expression of E-cad and the behavior of micrometastases in EGC, we examined the E-cad tissue status immunohistochemically. We found 
Table 4. Association between characteristics of lymph node metastases and loss of expression of E-cadherin in the 135 patients with EGC examined for E-cadherin expression

\begin{tabular}{lccc}
\hline Lymph node involvement & $\begin{array}{c}\text { No. of } \\
\text { cases }\end{array}$ & $\begin{array}{c}\text { Loss of expression of } \\
\text { E-cadherin }(\%)\end{array}$ & $P$ \\
\hline $\begin{array}{l}\text { Features of node involvement } \\
\text { Negative }\end{array}$ & 98 & $47(48)$ & \\
$\quad$ Single or scattered cells & 26 & $19(73)$ & 0.0008 \\
$\quad$ Clusters of cells & 11 & $11(100)$ & \\
Location of node involvement & & $47(48)$ & \\
$\quad$ Negative & 98 & $16(73)$ & \\
$\quad$ Group 1 nodes only & 22 & $14(93)$ & 0.0011 \\
$\quad$ Group 2 or 3 nodes & 15 & $47(48)$ & \\
No. of CK-positive nodes & 98 & $9(53)$ & \\
$\quad$ Negative & 13 & $7(100)$ & \\
$\quad$ One node & 17 & & \\
$\quad$ Two-three nodes & 7 & & \\
Four or more nodes & & & \\
\hline
\end{tabular}

Survival rate

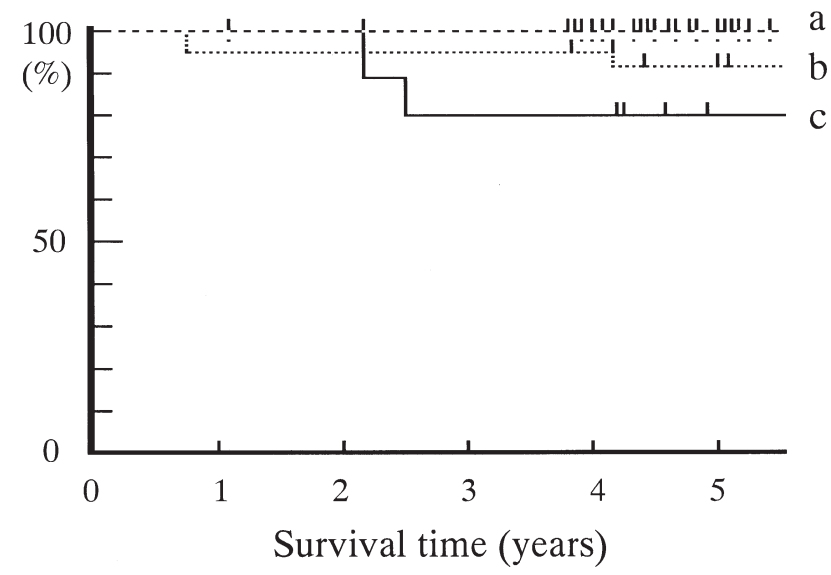

Fig. 3. Five-year survival curves of 162 patients with early gastric cancer (EGC). $a$ The 5-year survival of the 119 patients with no lymph node metastases $(100 \%) b$ The 5-year survival of the 32 patients with micrometastases in lymph nodes as detected by CK immunostaining $(93.6 \%) c$ The 5-year survival of the 11 patients with metastases in lymph nodes as detected by $\mathrm{H} \& \mathrm{E}$ staining $(81.8 \%)$. Generalized Wilcoxon test: $a$ vs $b, P<0.01 ; a$ vs $c, P<0.01$

that E-cad was strongly expressed, without exception, in the cell-cell boundaries of normal gastric mucosa adjacent to the primary tumor. Reduced expression of Ecad was found in $57 \%$ of our patients with EGC, a finding that strongly agrees with that in a report by Bolk et al. [28]. There was a tendency towards loss of expression of E-cad in tumors that were poorly differentiated, deeply invaded, or more than $1.0 \mathrm{~cm}$ in diameter, and in tumors with lymphatic or vascular invasion, and all of these factors were found to be strongly associated with lymph node metastasis as detected by CK immuno- staining. Markedly reduced expression of E-cad was found in 40 of 45 patients with poorly differentiated cancers. Poorly differentiated cancers and tumors with loss of expression of E-cad were also found to be more likely to metastasize to the extra-perigastric lymph nodes with a large number of clustered cancer cells. Moreover, $81 \%$ of the patients with lymph node involvement detected by CK immunostaining were found to have loss of expression of E-cad. Thus, reduced expression of E-cad would appear to lead to loss of its invasion-suppressor function, and subsequent loss of tumor differentiation, promoting cancer metastasis to the lymph nodes [25,27]. These findings, together with our results, suggest that poorly differentiated tumors with loss of expression of E-cadherin seem to constitute a very important risk factor for predicting lymph node involvement in EGC.

It has been reported that abnormal expression of Ecad contributes to gastric cancer metastases $[29,30]$. In the present study, only a single cancer cell or scattered cancer cells in the lymph nodes were detected in $65 \%$ of the 43 patients with CK-positive lymph nodes. It is still unclear whether these single or scattered cancer cells remain alive in the lymph node, or if they are destroyed or removed by the immune response of the host [12]. It was interesting to note, however, that $73 \%$ of these patients with only single or scattered cancer cells in the lymph node showed a marked loss of expression of Ecad in their primary tumor. Multivariate analysis showed that loss of expression of E-cad was an independent risk factor for these micrometastases with only single or scattered cancer cells in the lymph nodes. Survival analysis indicated that the outcome of these patients with micrometastases in the lymph nodes was significantly less favorable than outcome in patients 
Table 5. Logistic regression multivariate analysis of risk factors associated with lymph node metastasis detected by CK immunostaining

\begin{tabular}{lcc}
\hline Variables & Regression coefficient & $P$ \\
\hline Tumor size & 0.005 & 0.7597 \\
Macroscopic type (elevated, depressed) & 0.061 & 0.4887 \\
Ulceration (negative, positive) & 0.123 & 0.2843 \\
Histological type (differentiated, & 0.107 & 0.2144 \\
$\quad$ undifferentiated) & 0.100 & 0.2242 \\
Depth of invasion (mucosal, submucosal) & 0.394 & 0.0098 \\
Lymphatic invasion (negative, positive) & 0.041 & 0.7746 \\
Vascular invasion (negative, positive) & 0.222 & 0.0061 \\
\hline Expression of E-cadherin (normal, abnormal)
\end{tabular}

${ }^{a}$ See text for definition

with no such micrometatases. Therefore, it can be concluded that tumors with micrometatases of only a single or a few scattered cancer cells in the lymph node may already have potential metastatic activity, and these micrometastases may have the same consequence as clustered cancer cells in the lymph node, i.e., a reduced patient survival rate.

In recent years, limited surgery has been performed for the treatment of EGC. Based on our observations, we suggest the following possibilities: an endoscopic mucosal resection (EMR) or laparoscopic wedge resection without lymphadenectomy may be feasible for an $\mathrm{m}$-cancer of less than 1.0-cm superficial diameter, because lymph node involvement is extremely rare in these tumors; a limited perigastric lymph node dissection can be performed for an m-cancer that is 1.0 to $2.0 \mathrm{~cm}$ in diameter, because these tumors rarely metastasize to the extra-perigastric lymph nodes; a radical D2 lymphadenectomy is needed for an m-cancer that is more than $2.0 \mathrm{~cm}$ in diameter, and for all sm-cancers, because these tumors have the potential for extraperigastric lymph node involvement. Preoperative E-cadherin immunostaining and determination of histology from an endoscopic biopsy sample may be helpful for the prediction of lymph node involvement.

\section{References}

1. Japanese Research Society for Gastric Cancer. The general rules for gastric cancer study in surgery and pathology. Jpn J Surg 1981;11:127-45.

2. Maehara Y, Orita H, Okuyama T, Moriguchi S, Tsujitani S, Korenaga D, et al. Predictors of lymph node metastasis in early gastric cancer. Br J Surg 1992;79:245-7.

3. Yokota T, Saito T, Teshima S, Kikuchi S, Kunii Y, Yamauchi H. Lymph node metastasis in early gastric cancer: how can surgeons perform limited surgery? Int Surg 1998;83:287-90.

4. Ishigami S, Natsugoe S, Hokita S, Tokushige M, Saihara T, Watanabe $\mathrm{T}$, et al. Carcinomatous lymphatic invasion in early gastric cancer invading into the submucosa. Ann Surg Oncol 1999;6:286-9.

5. Baba H, Maehara Y, Okuyama H, Orita H, Anai H, Akazawa K, et al. Lymph node metastasis and macroscopic features in early gastric cancer. Hepatogastroenterology 1994;41:380-3.
6. Isozaki H, Tanaka N, Okajima K. General and specific prognostic factors of early gastric carcinoma treated with curative surgery. Hepatogastroenterology 1999;46:1800-8.

7. Sano T, Kobori O, Muto T. Lymph node metastasis from early gastric cancer: endoscopic resection of tumor. Br J Surg 1992;79: 241-4.

8. Yamao Y, Shirao K, Ono H, Kondo H, Saito D, Yamaguchi H, et al. Risk factors for lymph node metastasis from intramucosal gastric carcinoma. Cancer 1996;77:602-6.

9. Koufuji K, Takeda J, Toyonaga A, Yoshihara S, Tanaka Y, Ohta $\mathrm{J}$, et al. Early gastric cancer and lymph node metastasis. Kurume Med J 1997;44:157-64.

10. Shen KH, Wu CW, Lo SS, Hsieh MC, Hsia CY, Chiang SC, et al. Factors correlated with the number of metastatic lymph nodes in gastric cancer. Am J Gastroenterol 1999;94:104-8.

11. Seto Y, Nagawa H, Muto T. Impact of lymph node metastasis on survival with early gastric cancer. World J Surg 1997;21:186-90.

12. Ishida K, Katsuyama T, Sugiyama A, Kawasaki S. Immunohistochemical evaluation of lymph node micrometastases from gastric carcinomas. Cancer 1997;79:1069-76.

13. Maehara Y, Oshiro T, Endo K, Baba H, Oda S, Ichiyoshi Y, et al. Clinical significance of occult micrometastasis in lymph nodes from patients with early gastric cancer who died of recurrence. Surgery 1996;119:397-402.

14. Cai J, Ikeguchi M, Maeta M, Kaibara N, Sakatani T. Clinicopahtological value of immunohistochemical detection of occult involvement in pT3N0 gastric cancer. Gastric Cancer 1999; 2:95-100.

15. Yanemura Y, Ninomiya I, Kaji M, Sujiyama K, Fujimura T, Tsuchihara K, et al. Decreased E-cadherin expression correlates with poor survival in patients with gastric cancer. Anal Cell Pathol 1995;8:177-90.

16. Sasaki M, Watanabe H, Jass JR, Ajioka Y, Kobayashi M, Matsuda K, et al. Occult lymph node metastases detected by cytokeratin immuno-histochemistry predict recurrence in "node negative" colorectal cancer. J Gastroenterol 1997;32:758-64.

17. Hsu SM, Raine L, Fanger H. The use of avidin-biotin-peroxidase complex $(\mathrm{ABC})$ in immunoperoxidase techniques; a comparison between $\mathrm{ABC}$ and unlabeled antibody procedures. J Histochem Cytochem 1985;19:577-80.

18. Jawhari A, Jordan S, Poole S, Browne P, Pignatelli M, Farthing MJG. Abnormal immunoreactivity of the E-cadherin-catenin complex in gastric carcinoma: relationship with patient survival. Gastroenterology 1997;112:46-54.

19. Torii A, Sakai M, Inoue K, Yamabe H, Ueda, Okuma M. A clinicopathological analysis of early gastric cancer: retrospective study with special reference to lymph node metastasis. Cancer Detect Prev 1994;18:437-41.

20. Yasuda K, Shiraiahi N, Suematsu T, Yamaguchi K, Adachi Y, Kitano S. Rate of detection of lymph node metastasis is correlated with the depth of submucosal invasion in early stage gastric carcinoma. Cancer 1999;85:2119-23. 
21. Renaldi R, Santinelli A, Verdolini R, Rezai B, Mannello B, Bearzi I. Long-term follow-up in early gastric cancer: evaluation of prognostic factors. J Pathol 1995 177:343-51.

22. Morita M, Baba H, Fukuda T, Taketomi A, Kohnoe S, Seo Y, et al. Submucosal gastric cancer with lymph node metastasis. J Surg Oncol 1998;68:5-10.

23. Tsuchiya A, Kikuchi Y, Ando Y, Yoshida T, Abe R. Lymph node metastases in gastric cancer invading the submucosal layer. Eur J Surg Oncol 1995;21:248-50.

24. Lehnert T, Erlandson RA, Decosse JJ. Lymph and blood capillaries of the human gastric mucosae. A morphologic basis for metastasis in early gastric carcinoma. Gastroenterology 1985;89: 939-50.

25. Mayer B, Johnson JP, Leitl F, Jauch KW, Heiss MM, Schildberg $\mathrm{FW}$, et al. E-cadherin expression in primary and metastatic gastric cancer: down-regulation correlates with cellular dedifferentiation and glandular disintegration. Cancer Res 1993;53:1690-5.
26. Shino $\mathrm{Y}$, Watanabe A, Yamada $\mathrm{Y}$, Tanase M, Yamada T, Matsuda M, et al. Clinicopathologic evaluation of immunohistochemical E-cadherin expression in human gastric carcinomas. Cancer 1995;76:2193-201.

27. Shiozaki H, Oka H, Inoue M, Tamura S, Monden M. E-cadherin mediated adhesion system in cancer cells. Cancer 1996;77(Suppl): 1605-13.

28. Bolk P, Craanen ME, Dekker W, Tytgat GNJ. Loss of E-cadherin expression in early gastric cancer. Histopathology 1999;34:410-5.

29. Shun CT, Wu MS, Lin JT, Wang HP, Houng RL, Lee WJ, et al. An immunohistochemical study of E-cadherin expression with correlations to clinicopathological features in gastric cancer. Hepatogastroenterology 1998;45:944-9.

30. Ramesh S, Nash J, McCulloch PG. Reduction in membranous expression of $\beta$-catenin and increased cytoplasmic E-cadherin expression predict poor survival in gastric cancer. $\mathrm{Br} \mathrm{J}$ Cancer 1999;81:1392-7. 